\title{
Esclerosis Tuberosa asociada a Síndrome Nefrótico y falla renal aguda
}

\author{
Gabriela Santos Revilla ', Kevin Schreiber Álvarez ', Aníbal Manrique Acha ', Luis Miguel Orellana Solís ', Josué Bernardo Holguín ', \\ David Loja Oropeza ${ }^{2}$
}

\section{RESUMEN}

El complejo de esclerosis tuberosa es una facomatosis, caracterizada por lesiones cutáneas, epilepsia y retardo mental; de variable afectación a: cerebro, riñones, corazón y otros órganos. De carácter autosómico dominante, existe entre un 60 a 70\% de mutaciones de novo. Las lesiones renales se pueden identificar hasta en un $57.5 \%$ de pacientes.

Se reporta el caso de un paciente varón de 20 años de edad en el Hospital Nacional Arzobispo Loayza. Se presentó con angiofibromas cutáneos, placas de Chagrin, calcificaciones subependimarias, angiomiolipomas renales y leve retardo mental. Con un intenso síndrome nefrótico asociado a insuficiencia renal aguda. Sin antecedentes familiares.Se revisó la literatura y las diversas formas de presentación clínica-patológica de la esclerosis tuberosa. (Horiz Med 2014; 14(1): 54-58)

Palabrasclave: esclerosistuberosa, manifestaciones renales, manifestacionesneurológicas, manifestaciones dermatológicas. (Fuente: DeCS BIREME).

\section{Tuberous Sclerosis complex associated to Nephrotic Syndrome and Acute Kidney Injury}

\section{ABSTRACT}

The tuberous sclerosis complex is a phakomatosis, characterized by cutaneous lesions, epilepsy and mental retardation; with variable affectation in brain, kidneys, heart and other organs. With dominant autosomic character, there are between 60 to $70 \%$ de novo mutations. Renal lesions can be identified in up to $57.5 \%$ of patients.

We report the case of a 20 years old male patient from the Hospital NacionalArzobispoLoayza. He presents cuteanousangiofibromas, Shagreen patches, subependymal calcifications, renal angiomyolipomas and mild mental retardation; along with an intense nephrotic syndrome associated with acute renal injury. No family history of importance. Literature is revised along with the diverse forms of clinical-pathologic presentations of tuberous sclerosis complex.

(Horiz Med 2014; 14(1): 54-58)

Key words: tuberous sclerosis complex, renal manifestations, neurological manifestations, skin manifestations (Source: MeSH NLM).

\footnotetext{
${ }^{1}$ Estudiantes de la Facultad de Medicina Humana - USMP. Lima-Perú

${ }^{2}$ Departamento de Medicina Interna. Hospital Nacional Arzobispo Loayza. Lima-Perú
} 


\section{INTRODUCCIÓN}

La esclerosis tuberosa (ET, O TSC por Tuberous Sclerosis Complex), también Ilamada Enfermedad de Bourneville, es una enfermedad perteneciente a las denominadas facomatosis (síndromes neurocutáneos como la enfermedad de Von Recklinghausen, el síndrome de Sturge Weber - Angiomatosis encéfalofacial y el síndrome de Von Hippel Lindau) caracterizadas por lesiones cutáneas, epilepsias y retardo mental (Triada de Vogt).

Descrita por primera vez en 1880 , es un desorden genético, de carácter autosómico dominante, que afecta órganos como el cerebro, los riñones, el corazón, los ojos y otros (1).

En Latinoamérica, no existe la suficiente documentación respecto a la presentación de la ET. En Brasil, se halló una incidencia de 1 por 10.000 a 50.000 nacidos vivos; con mutaciones de novo en el $85 \%$ de los casos.

En el Perú, se han descrito 19 casos de hallazgo pediátrico de ET en el Hospital Nacional Cayetano Heredia, dentro de los cuales el $88 \%$ debutaron con convulsiones antes de los 11 meses (2).

Existen pocas referencias sobre esta enfermedad en nuestro país, por lo que reportamos el presente caso observado en el HN A. Loayza y revisamos la literatura.

\section{CASO CLÍNICO}

Paciente varón de 20 años de edad, procedente del Cuzco, que inicia enfermedad con edema generalizado, distensión y un leve dolor abdominal.

Dos meses antes del ingreso al hospital, el paciente fue atendido en su lugar de origen y hospitalizado alrededor de 1 mes. Fue diagnosticado de Esclerosis tuberosa por lo que es referido para estudio especializado. Presentó apetito disminuido, sed aumentada y diuresis disminuida. Su peso antes de la anasarca fue de 58 kilos, llegando a 68 kilos al momento de su ingreso. Con antecedente de pobreza extrema, con inmunizaciones incompletas, nacido de parto eutócico domiciliario. Diagnosticado de retardo mental leve, el 17 de agosto del 2012. Su padre, madre y hermanos son aparentemente sanos.

El paciente refirió: epistaxis frecuentes, edemas, vómitos y gonalgiahasta el momento del ingreso. Los familiares refirieron que una vez tuvo pérdida transitoria de la conciencia.

Examen físico: Facie edematosa, con edema palpebral y en anasarca; con múltiples lesiones papulares rojizas, en el rostro, tórax y abdomen; y lesiones elevadas hipopigmentadas en tórax y glúteos (Figura 1).

Se tomó biopsia de la piel del rostro por punch, siendo el diagnóstico patológico de angiofibroma (Figura 2).
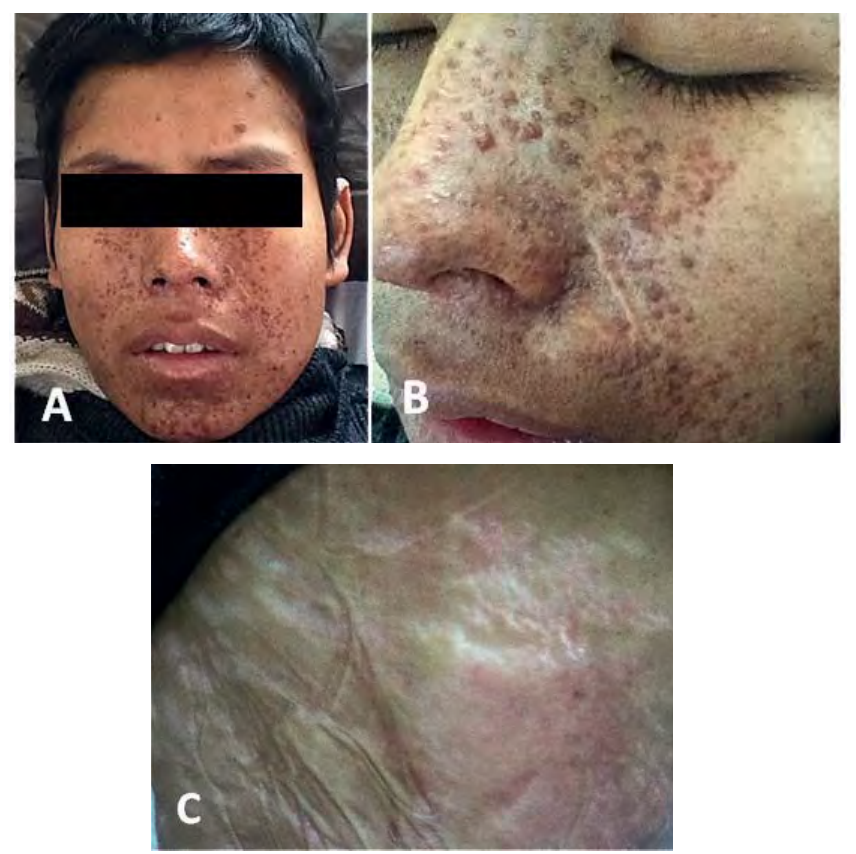

Figura 1. Múltiples lesiones papulares rojizas, en rostro (A) (B). Lesiones hipopigmentadas elevadas, en espalda y glúteos, compatibles con placas de Chagrin (C)

En el examen de tórax y pulmones, se encontraron signos compatibles con derrame pleural izquierdo, confirmado por radiografía simple. El estudio del líquido pleural evidenció un ADA de $15 \mathrm{U} / \mathrm{L}$.

Se evidenció leve retardo mental, con capacidad de atención alterada. 


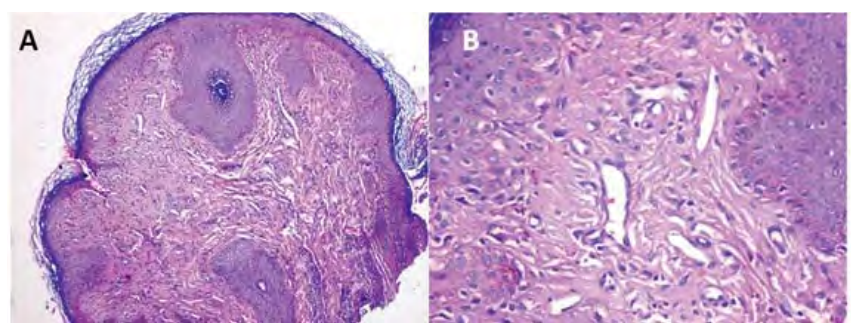

Figura 2. Angiofibroma diagnosticado por biopsia de piel. Se observan pápulas con vasos sanguíneos, rodeados de células estromales fibrosas y algunas zonas hemorrágicas. Aumento a 20x (A) y 40x (B), tinción de Hematoxilina/Eosina.

Se realizó una Tomografía Axial Multicorte (TEM) cerebral, la cual mostró presencia de calcificaciones subependimarias adyacentes a periferie ventricular, la más grande de $8 \times 5 \mathrm{~mm}$, adyacente al ventrículo lateral izquierdo.

\section{Estudio ecográfico abdominal completo:} esteatosis hepática en grado III, signos de nefropatía intersticial bilateral, ascitis moderada, riñones con ecogenicidad aumentada, con imágenes hiperecogénicas nodulares menores de $1 \mathrm{~mm}$, y un quiste de $18 \mathrm{~mm}$ en polo inferior izquierdo. Se realizó una biopsia a cielo abierto del riñón derecho, hallándose angiomiolipomas y glomeruloesclerosis focal y segmentaria (Figura 3).

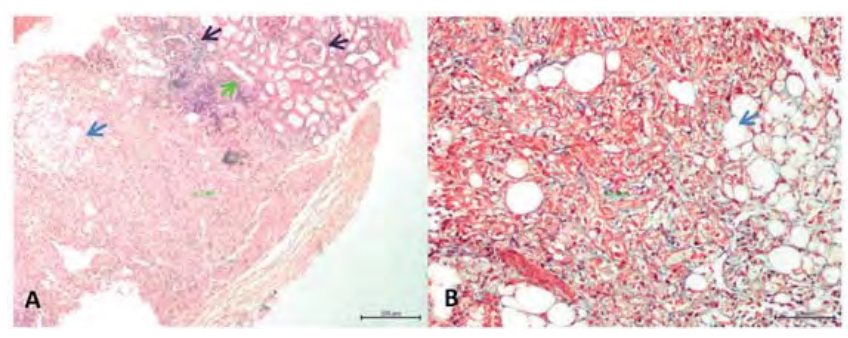

Figura 3. Angiofibroma diagnosticado por biopsia de piel. Se observan pápulas con vasos sanguíneos, rodeados de células estromales fibrosas y algunas zonas hemorrágicas. Aumento a 20x (A) y 40x (B), tinción de Hematoxilina/Eosina.

Estudio bioquímico: proteinuria (1987mg/24h, vol: $540 \mathrm{ml}$ ), hipoalbuminemia sostenida, hipertrigliceridemia $\quad(384 \quad \mathrm{mg} / \mathrm{dl}) \quad$ e hipercolesterolemia (350 mg/dl); así como falla renal (oscilante desde 47,4 a 25,48ml/min $/ 1.73$ $\mathrm{m} 2)$.

\section{DISCUSIÓN}

La esclerosis tuberosa, es considerada la segunda en frecuencia en facomatosis, superada por la neurofibromatosis. Se calcula que entre 10 y 14 en 100,000 mil personas la padecen en todo el mundo. Se reporta un caso entre 6000 recién nacidos por año (3)

La ET se presenta cuando los genes TSC1 (en el locus cromosomal 9q34.3) o TSC2 (16p13.3) son inactivados. Estos genes codifican las proteínas hamartina y tuberina respectivamente, las cuales parecen intervenir en la supresión de tumores al regular la proliferación celular (4).

Las células altamente especializadas en las lesiones pueden alcanzar un tamaño gigante, denotando el carácter blastomatoso de la ET y sugiriendo que algún factor inhibitorio del crecimiento está fallando en la vida embriogénica; induciendo la hiperplasia y la hipertrofia de células bien diferenciadas, lo que caracteriza los signos (5).

Los criterios diagnósticos han sido actualizados en el año 2012 por la Conferencia Internacional para el Consenso del Complejo de Esclerosis Tuberosa, y comprenden 11 criterios mayores y 6 criterios menores; que representan medios más actualizados y de mejor evidencia para el diagnóstico de la TSC (1).

Las mutaciones en los genes TSC1 y TSC2 presentan diferente prevalencia en aparición de casos familiares y esporádicos, siendo la mutación TSC1 significativamente menos relacionada con los casos de novo (6).

Se estima que existe significativamente mayor incidencia de patologías y características de severidad en aquellos pacientes con una mutación del TSC2, sean asociados al sistema nervioso, renal u otros órganos (7).

El paciente ingresa con un síndrome edematoso, más un síndrome dermatológico y retardo mental leve. El compromiso neurológico asociado a un síndrome dermatológico representa un tipo de facomatosis. 
Por el aparente compromiso renal asociado, se tendría como posibilidades al SVHL y a la ET, sin embargo, por el espectro autista presentado en el paciente, la ET queda como principal sospecha.

En el examen físico, la piel en tórax presenta placas de Chagrin; en el estudio de anatomía patológica la piel del rostro muestra angiofibromas y lesiones en confeti. En la TEM, se aprecian nódulos subependimarios; y la biopsia renal evidencia angiomiolipomas. Tendríamos entonces 3 criterios mayores y 1 menor, lo que configura el diagnóstico definitivo de la enfermedad de TSC. Hipotéticamente, se podría plantear que esta mutación de novo es del gen TSC2, dado lo florido de la clínica y los hallazgos de imágenes, que sugieren un cuadro de mayor severidad en el sistema nervioso, renal y piel; comparados con aquellos más leves y benignos de las mutaciones del gen TSC1 (7).

En cuanto a las implicaciones neurológicas en el TSC, se incluyen espasmos epilépticos infantiles, epilepsia refractaria, tumores cerebrales, trastornos neurocognitivos diversos y autismo (8).

En este caso, el paciente presentó trastornos en la conducta, con rasgos autistas, retraso mental leve y aparentemente no habría presentado crisis epilépticas, aunque sus familiares refieren que una vez tuvo pérdida transitoria de la conciencia.

Los nódulos subependimarios son lesiones hamartomatosas situadas en la superficie ependimaria de los ventrículos laterales y que en el $90 \%$ de los casos tienden a calcificarse, como ocurrió en este caso. Un 5-10\% de estas lesiones evolucionan hacia astrocitomassubependimarios de células gigantes (9).

Los angiofibromas faciales son tumoraciones benignas en la cara que tienden a aparecer en las mejillas y en la nariz. Su uso para el diagnóstico precoz de esclerosis tuberosa, tiende a ser muy escasa pues aparecen en la niñez tardía y solo entre un 70 a $80 \%$ de los casos (10).

Otras lesiones dermatológicas, ausentes en este caso, incluyen los tumores de Koenen (fibromas periungueales), la placa frontal, manchas hipomelanóticas lanceoladas en hoja de fresno, manchas en "confeti" y los fibromas gingivales (11). A pesar que la ET produce afectación renal, las complicaciones clínicas son raras (4). Sin embargo, se han reportado casos de complicaciones con falla renal crónica (12).

Las complicaciones asociadas a los quistes y angiomiolipomas son: insuficiencia renal y la hipertensión arterial secundarias a los efectos de masa así como al sangrado de los AML, llevando a hematuria similar al cólico nefrítico o hemorragia retroperitoneal (13).

La enfermedad quística renal y los angiomiolipomas son causas recurrentes de enfermedad crónica renal, siendo algunas veces indetectables por el estudio de imágenes (14).

Llama la atención la asociación de síndrome nefrótico a la ET, evento no descrito hasta ahora. En la histopatología se evidenció gloméruloesclerosis focal y segmentaria, trastorno cuya etiología podría ser secundaria de causa genética, con una mutación en los genes codificadores de proteínas de la membrana basal, diafragma hendido 0 en el podocito (gen ACTN4 y gen TRPC6), lo que constituiría un denominador común de cambios mutagénicos en varias áreas mostrando diversidad de hallazgos clínicos e histológicos (15).

Evoluciono con S. nefrótico que progresó a falla renal aguda sin requerimiento de diálisis y que se resolvió espontáneamente. No presentó síndrome nefrítico ni depleción de complemento.

En el Perú, existe insuficiente iniciativa en la investigación sobre la prevalencia de la Esclerosis Tuberosa, posiblemente desestimando características propias de la enfermedad; por lo cual se aconseja ampliar su estudio.

\section{Fuente de Financiamiento}

El presente trabajo fue financiado a través de recursos propios de los investigadores. 


\section{Conflictos de interés}

Los autores declaran no tener conflictos de interés en la publicación de este artículo.

\section{AGRADECIMIENTOS}

A los doctores: Carmen Asato Higa (Patología Clínica, Laboratorios Integrados, Lima-Perú), César Chian García (Departamento de Patología del HN A. Loayza) y José Caballero Silva (Departamento de Medicina Interna del HN A. Loayza) por su colaboración en la obtención de las microfotografías patológicas y la prestación de sus servicios para la realización del presente reporte.

\section{REFERENCIAS BIBLIOGRÁFICAS}

1. Northrup $\mathrm{H}$, Krueger DA, International Tuberous Sclerosis Complex Consensus Group. Tuberous sclerosis complex diagnostic criteria update: recommendations of the 2012 international tuberous sclerosis complex consensus conference. Pediatr Neurol 2013; 49(4):243-54

2. Medina-Malo C, Carreño O, Vélez A, Lizcano Luis A, et al. Complejo esclerosis tuberosa. Acta Neurol Colomb. 2012 Mar; 28(1): 11-23.

3. Fernández Concepción Otman, Gómez García Ariel, Sardiñaz Hernández Norberto.

Esclerosis Tuberosa. Revisión. Rev Cubana Pediatr 1999; (3): 160-167

4. Rakowski SK, Winterkorn EB, Paul E, Steele DJR, Halpern EF and Thiele EA. Renal manifestations of tuberous sclerosis complex: Incidence, prognosis, and predictive factors. Kidney International. 2006.

5. Velasco María del Carmen, Chávez Olvera José Luis. Esclerosis tuberosa. Presentación de un caso y revisión de la literatura. Rev Mex Med Física y Rehabilitación 2001; 13: 89-98

6. Alistair C. Jones, Claire E. Daniells, Russell G. Snell, Maria Tachataki,Shelley A. et al. Molecular Genetic and Phenotypic Analysis Reveals Differences between TSCland

TSC2 Associated Familial and Sporadic Tuberous Sclerosis. Hum Mol Genet 1997; 6(12): 2155-2161
7. Camposano SE, Greenberg E, et al. Distinct clinical characteristics of Tuberous Sclerosis Complex patients with no mutation identified. Annals of Human Genetics 2009 73,141-146

8. García-Peñas Juan José, Carreras-Sáez Inmaculada. Autismo, epilepsia y esclerosis tuberosa: un modelo de conexión funcional a través de la vía mTOR. Rev Neurol 2013; 56 (Supl. 1):S153-S161]

9. Umeoka S, Koyama T, Miki Y, et al. Pictorial review of tuberous sclerosis in various organs. Radiographics 2008; 28: e32

10. Hyman $\mathrm{MH}$, Whittemore $\mathrm{VH}$. National Institutes of Health Consensus Conference: Tuberous sclerosis complex. ArchNeurol 2000; 57:662-665

11. Monteagudo B, De las Heras C, et al. Placas de chagrin. An Pediatr 2007; 66: 4.

12. Dhakal M, Dhakal OP, Bhandari D. Polycystic kidney disease and chronic renal failure in tuberous sclerosis.BMJ Case Rep 2013; Oct 2.

13. López Buitrago, Piedecuasa Selfa, et al. Hipertensión e insuficiencia renal ligadas al complejo de esclerosis tuberosa. Hipert Riesgo Vasc 2011;28(5-6):204-207.

14. Dixon BP, Hulbert JC, Bissler JJ.

Tuberous Sclerosis Complex Renal Disease.

Nephron Exp Nephrol 2011; 118:e15-e20

15. Restrepo Cesar A. Glomeruloesclerosis focal y segmentaria. Asoc Colomb Nefrol Hipert Art 2008; $1: 1$

\section{Correspondencia:}

Gabriela Santos Revilla

Dirección: Av. Alfonso Ugarte s/n, Lima-Perú

Teléfono: 998068006

Correo electrónico: gabriela.sr92@hotmail.com 